\title{
Improving rice grain length through updating the GS3 locus of an elite variety Kongyu 131
}

Jianzong Nan ${ }^{1,2}$, Xiaomin Feng ${ }^{1,2}$, Chen Wang ${ }^{1,2}$, Xiaohui Zhang ${ }^{1,2}$, Rongsheng Wang ${ }^{1,2}$, Jiaxin Liu ${ }^{1}$, Qingbo Yuan ${ }^{1}$, Guoqiang Jiang ${ }^{1}$ and Shaoyang $\operatorname{Lin}^{1,2^{*}}$ (1)

\begin{abstract}
Background: Traditional crop breeding has made significant achievement meet food needs worldwide. However, the way has some inevitable problems including time-consuming, laborious, low predictability and reproducibility. In this study, we updated the GRAIN SIZE 3 (GS3) locus to improve the grain length of a major cultivate variety of Kongyu 131 at Heilongjiang Province, the northernmost region of China. High-resolution melting (HRM) analysis is used for single nucleotide polymorphism (SNP) genotyping.

Results: The improved line introgressed about $117 \mathrm{~kb}$ segment including gs3 allele from donor GKBR by using five SNP markers designed within and without GS3 locus, and the background recovery ratio of the recurrent parent genome is about $99.55 \%$ that are detected by 219 SNP markers evenly distributed on the 12 chromosomes. The field trial indicates that grain length, 100-grain weight and total grain weight per plant of the improved line raised by $12.05 \%, 16.30 \%$ and $4.47 \%$, respectively, compared with Kongyu 131.
\end{abstract}

Conclusions: This result demonstrates that update the GS3 locus is a feasible and efficient and accurate way can be applied to improve grain size of rice.

Keywords: Rice, Kongyu 131, GS3, SNP, HRM, Breeding

\section{Background}

It is a great challenge enhancing production of crops to supply the increasing demand continuously; on the one hand, the arable land is decreasing with the development of urbanization, on the other hand, the production of crop is influenced inevitably by some environment factors of global warming more and more (Takeda and Matsuoka 2008). Some investigations indicate that total production of crops has growing slowly at some district, even decreased at other area (Ray et al. 2012). Therefore, it is an urgent challenge to supply the demand of accumulative population by increasing crop productivity.

Rice is a staple crop cultivated extensively, and it supply over half diets of population all around the world. Also rice is a model monocotyledon with a small

\footnotetext{
* Correspondence: sylin@genetics.ac.cn

${ }^{1}$ State Key Laboratory of Plant Genomics and National Plant Gene Research Center, Institute of Genetics and Developmental Biology, Chinese Academy of Sciences, Beijing 100101, China

${ }^{2}$ University of Chinese Academy of Sciences, Beijing 100039, China
}

genome and complete genome sequence and a large number of useful resources. It is considered that the production of per plant determined by panicle numbers per plant, grain numbers per panicle, grain weight and filling (Sakamoto and Matsuoka 2008; Xing and Zhang 2010). Grain weight is a vital factor influences production of rice; therefore, it is a potential way to improve production of rice by updating the locus associated with grain weight. And also the long-grain shape has been a new favored quality trait in the breeding of japonica rice in recent years (Haixiang and Qian 2017). Many grain size related genes in rice were identified in the past decade (Huang et al. 2013; Miura et al. 2011; Wang and Li 2011; Xing and Zhang 2010; Zuo and Li 2013). GS3 is the first identified and molecularly characterized QTL controls grain size (Fan et al. 2006; Mao et al. 2010).

Traditional breeding almost depends on field selection by experience of breeders to improve crop variety. However, the selection has many inevitable defects including time- 
consuming and laborious, low predictability and reproducibility. Using markers, linkage with the target gene on the basis of DNA sequence, has greatly facilitate accurate and diminish time cost of selection, namely marker-assisted selection (MAS) (Knapp 1998). However, unavoidable recombination limited precision of selection between target gene and markers (Andersen and Lubberstedt 2003). Meanwhile, traditional breeding selection with MAS has no consideration of whole genome elimination of non-target fragments except for selection target phenotype. Therefore, it is still uncertain that the effects of non-target segments and reserve background genome of recurrent parent at the most extent.

The concept of design breeding was proposed by two scientists from Israeli (Peleman and van der Voort 2003). Now, it is possible that breeders can improve crop combining or incorporating beneficial genes using a great amount of genome and functional gene information with development of sequencing and molecular method technology (Huang et al. 2013; James et al. 2003; Miura et al. 2011; Sakamoto and Matsuoka 2008; Wang and Li 2008, 2011; Xing and Zhang 2010; Zhou et al. 2013; Zuo and Li 2013).

Kongyu 131 is a small grain variety, which once was a major cultivar in the Heilongjiang province of northeast China. This variety belong to a high-latitude japonica, which has early maturity, strong tillering strength and resist lodging, and average production about $7684.5 \mathrm{~kg}$ per hectare. In 2005, the planting area of Kongyu 131 reached 770,000 ha, accounting for more than half of the total rice planting area. And the planting area of Kongyu 131 reached more than 560,000 ha from 2006 to 2011. It is estimated that the planting area of Kongyu 131 still have least 400,000 ha last few years.

To improve the grain size of the elite variety Kongyu 131, we carried out QTL analysis with grain size and detected desirable allele around the GS3 locus. Therefore we used the Update approach (Feng et al. 2017) to improve the grain size of a major cultivates variety of japonica Kongyu131. We developed SNP markers between Kongyu 131 and GKBR based on SNPs evenly distributed on rice 12 chromosomes. And also we designed five SNP markers at GS3 locus (SNP3) including its upstream (SNP1 and SNP2) and downstream (SNP4 and SNP5) on the basis of sequence difference between Kongyu 131 and GKBR. SNP markers were identified by using HRM analysis (Wittwer et al. 2003). Finally, we selected a line, named improved line, which segment of gs 3 allele about $117 \mathrm{~kb}$ is from donor GKBR and the background recovery ratio of Kongyu 131's about 99.55\%. The field trial indicates that grain length and 100-grain weight of the improved line significantly heightened compared with Kongyu 131, and total grain weight per plant also increased. This result demonstrates that the Update approach is an efficient and accurate way can be utilized to improve grain size of rice varieties.

\section{Results \\ QTL analysis detected a grain length related locus nearby GS3}

To detect allele can increase Kongyu 131's grain length, we measured grain length and analyzed genotype with an $\mathrm{F}_{2}$ populations constructed by Kongyu 131 and GKBR, and made QTL analysis according to Mapmaker/ QTL 1.1b (Lincoln et al. 1993). We detected a QTL related to grain length on the chromosome 3 (Fig. 1a), nearby GS3 locus, presumably this QTL is GS3 locus. And also we noticed another QTL on the chromosome 7, but we found this QTL from donor's allele not only related with grain length but also delay heading date. Given the delayed heading date cannot fully mature in
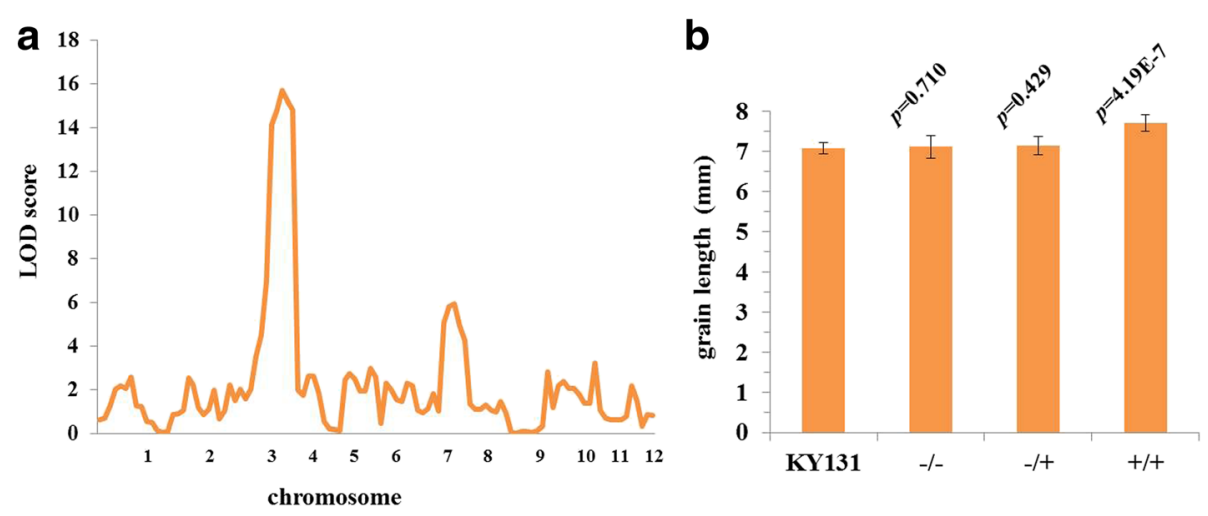

Fig. 1 QTL analysis detected a locus related grain length nearby the GS3 locus. a QTL analysis using $F_{2}$ population with 136 individuals and 123 SNP markers. $\mathbf{b}$ the grain length is significantly increased with the donor GKBR allele at the GS3 locus. $n=10$. The values represent the mean \pm s.d. $p$ values from the student's t-test of the plant with different genotype at GS3 locus against Kongyu131 is indicated. KY131 is the abbreviation of Kongyu 131 

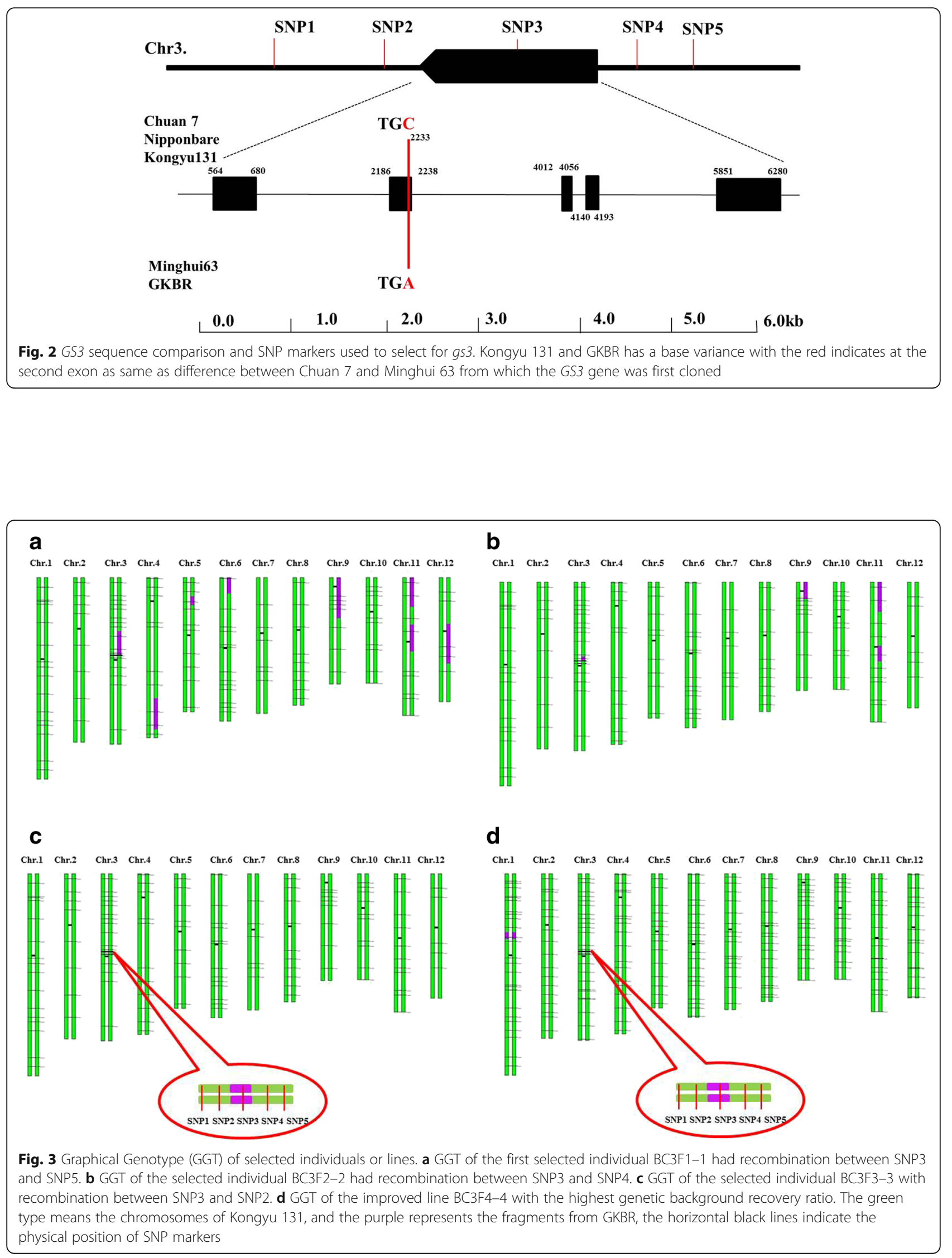
Heilongjiang, we only introgressed the QTL on the chromosome 3 in this study.

\section{Kongyu 131 with small grain allele and GKBR large grain allele at GS3 locus}

To identify whether donor GKBR has large grain allele at GS3 locus or not, we compared the coding DNA sequence (CDS) of GS3 gene between Kongyu 131 and GKBR using software of DNAMAN. We found a base difference at the second exon between Kongyu 131 and GKBR (Fig. 2). This variance at GS3 locus is same as the difference between a small grain cultivar, Chuan 7, and a large grain cultivar Minghui 63, which are two parents used to clone the GS3 for the first time. And also the researchers demonstrated that the variance of GS3 lead to premature termination of transcription in the large grain varieties (Fan et al. 2006; Mao et al. 2010). Therefore, we supposed that Kongyu 131 is the small grain allele and GKBR is the large grain allele at GS3 locus.

\section{Five SNP markers used to select for $g s 3$ allele from donor GKBR}

To select for gs3 allele from donor GKBR accurately, we developed a SNP marker, named SNP3 (Fig. 2), located within GS3 gene. And also we designed other four SNP markers- SNP1, SNP2, SNP4, SNP5 (Fig. 2)- located at upstream and downstream of GS3 gene, respectively, the physical distance about 1 Mega base pair (Mbp) between SNP1 and SNP5. These four markers mainly used to eliminate the drag of non-targeted segment.

\section{Genotype of an individual incorporated around $117 \mathrm{~kb}$ at GS3 locus from donor GKBR}

To acquire individuals incorporated the smallest segment of gs3 from donor GKBR. First, we selected 25 individuals with heterozygote genotype at SNP3 from the $\mathrm{BC}_{3} \mathrm{~F}_{1}$ population. And we found 8 of 25 individuals had recombination between SNP1 and SNP5. An individual was selected with highest genetic background recovery ratio from the 8 individuals using 134 SNP markers, named BC3F1-1 (Fig. 3a), which had recombination between SNP3 and SNP5. Second, we selected an individual, named BC3F2-2 (Fig. 3b), which had recombination between SNP3 and SNP4, from the progeny of BC3F1-1. Third, to get individuals had recombination between SNP3 and SNP1 or SNP2, we further selected from 400 progeny of BC3F2-2. Finally, we selected an individual with recombination between SNP3 and SNP2. We dubbed the selected individual BC3F3-3 (Fig. 3c), which incorporated about $117 \mathrm{~kb}$ homozygote segment from donor GKBR at GS3 locus.
The improved line with genetic background recovery ratio $99.55 \%$

To get the improved line with highest genetic background recovery ratio, we selected an individual named BC3F4-4 (Fig. 3d), which genetic background recovery ratio of Kongyu 131 is about 99.55\%, using 219 SNP markers evenly distributed on 12 chromosomes from the progeny of BC3F3-3. And also we found a non-target segment at chromosome 1 (Fig. 3d) according to those increased 85 markers, which had not been identified at BC3F1-1, BC3F2-2 and BC3F3-3.

\section{Grain length and weight of the improved line significantly increased}

To verify gs3's function of the improved line, we cultivated Kongyu 131 and the improved line 96 individuals with three replications at Heilongjiang, respectively. According to general management of trial plot, we investigated traits including grain length, grain width, 100-grain weight, plant height and total grain weight and so on (Table 1). The improved lines' grain length (Fig. 4b, Fig. 6a) and 100-grain weight (Fig. 4c) increased significantly compared with Kongyu 131, and total grain weight increased non-significant (Fig. 4d). We also found the number of panicle per plant of the improved lines decreased significantly (Fig. 4f). In some sense, this result can explain the reason why the improved lines' total grain weight increased non-significant while

Table 1 Agronomic performance of Kongyu 131 and its improved line BC4F4-4 in 2016 and 2017

\begin{tabular}{|c|c|c|c|c|}
\hline \multirow[t]{2}{*}{ Traits } & \multicolumn{2}{|l|}{2016} & \multicolumn{2}{|l|}{2017} \\
\hline & $\mathrm{BC} 3 \mathrm{~F} 4-4$ & KY 131 & $\mathrm{BC} 3 \mathrm{~F} 4-4$ & KY 131 \\
\hline$\overline{\mathrm{GL}}(\mathrm{mm})$ & $7.73 \pm 0.14^{*}$ & $6.90 \pm 0.11$ & $7.90 \pm 0.13^{*}$ & $6.94 \pm 0.09$ \\
\hline $\mathrm{GW}(\mathrm{mm})$ & $3.59 \pm 0.08$ & $3.53 \pm 0.05$ & $3.47 \pm 0.11$ & $3.44 \pm 0.03$ \\
\hline HGW(g) & $3.08 \pm 0.04^{*}$ & $2.65 \pm 0.05$ & $2.98 \pm 0.05^{*}$ & $2.73 \pm 0.06$ \\
\hline $\operatorname{TYP}(g)^{a}$ & $50.27 \pm 4.81$ & $48.11 \pm 7.7$ & $48.82 \pm 6.45$ & $48.39 \pm 5.87$ \\
\hline $\operatorname{TYP}(g)^{b}$ & $55.96 \pm 11.16^{*}$ & $40.82 \pm 4.31$ & - & - \\
\hline $\operatorname{TYP}(g)^{c}$ & - & - & $48.52 \pm 8.26$ & $48.06 \pm 3.79$ \\
\hline PNP & $27.4 \pm 2.87^{*}$ & $31.3 \pm 3.16$ & $24.13 \pm 1.73^{*}$ & $34 \pm 3.38$ \\
\hline GNP & $125.8 \pm 10.51$ & $116.8 \pm 23.5$ & $126.5 \pm 14.13$ & $116.38 \pm 8.42$ \\
\hline $\mathrm{PH}(\mathrm{cm})$ & $72.2 \pm 2.82$ & $71.45 \pm 1.06$ & $72.63 \pm 0.58^{*}$ & $67.60 \pm 1.75$ \\
\hline $\mathrm{PL}(\mathrm{cm})$ & $17.7 \pm 1.22$ & $17.58 \pm 1.74$ & $16.28 \pm 1.35$ & $16.06 \pm 0.63$ \\
\hline DTH & $107.25 \pm 2.06$ & $107.25 \pm 2.63$ & $102.50 \pm 1.64$ & $102.83 \pm 2.14$ \\
\hline
\end{tabular}

Data presented as the means with standard deviations were obtained from plants in a randomized complete block design with three replications under natural conditions at Jiamusi in 2016 and 2017. The planting density was $30 \mathrm{~cm} \times 20 \mathrm{~cm}$ and one plant per hill

GL Grain length, GW Grain width, HGW Hundred grain weight, TYP Total grain yield per plant, $P N P$ panicle number per plant, GNP grain number of main panicle, $P H$ plant height, $P L$ main panicle length, DTH Days to heading *represents significance at $p \leq 0.05$ based on Student's $t$-tests, $n=10$

${ }^{a}$ The planting density was $30 \mathrm{~cm} \times 20 \mathrm{~cm}$ and one plant per hill

${ }^{\text {b}}$ The planting density is $30 \mathrm{~cm} \times 20 \mathrm{~cm}$ and three to four plants per hill 'The planting density is $30 \mathrm{~cm} \times 14 \mathrm{~cm}$ and three to four plants per hill; indicates no data 


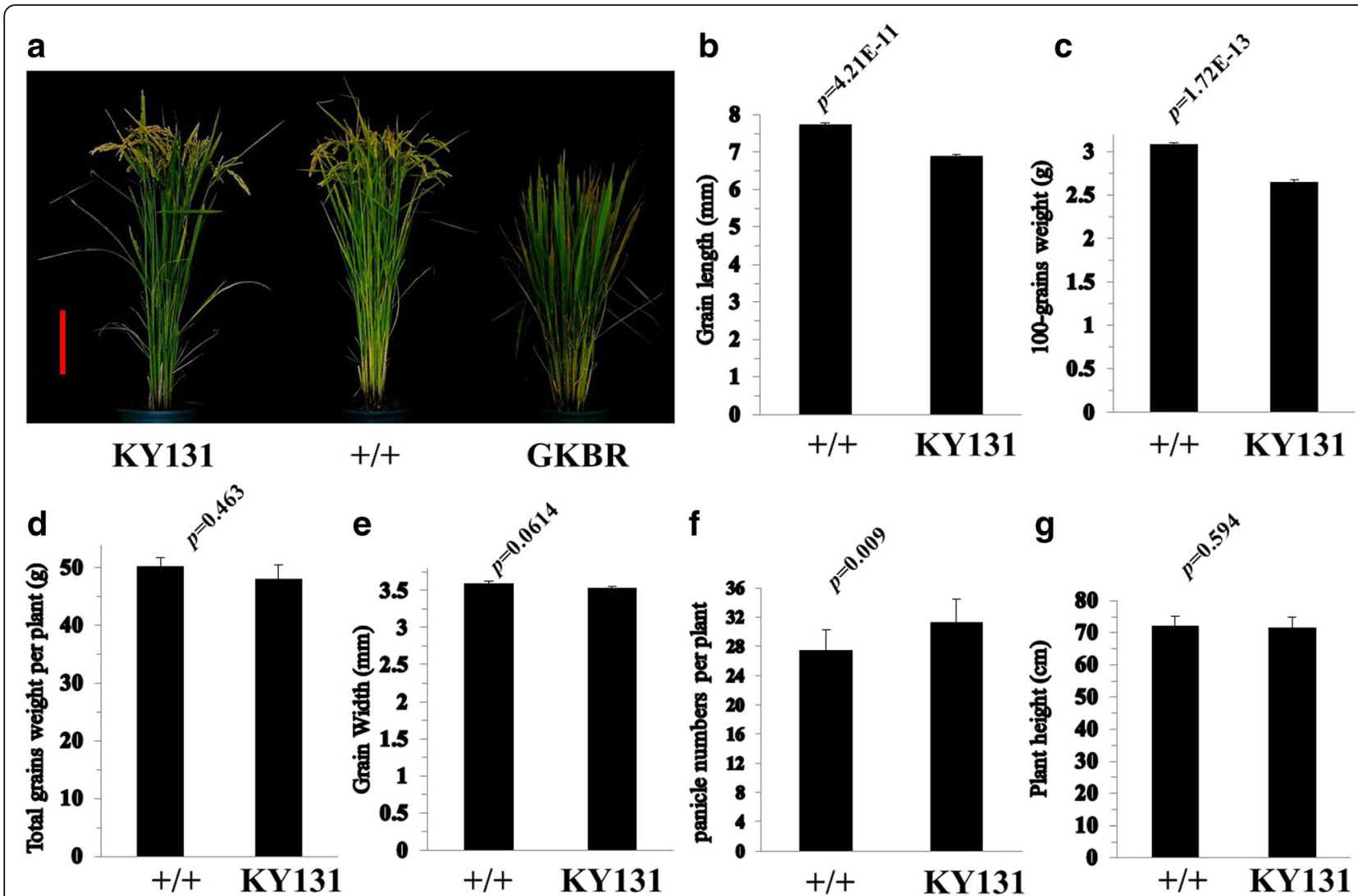

Fig. 4 Grain length and 100-grain weight significantly increased of the improved line compared with Kongyu 131. a Plant type of recurrent parent Kongyu 131, the improved line and the donor GKBR. Scale bar, $30 \mathrm{~cm}$. b-g Comparison of yield-related traits of Kongyu 131 and the improved line. For $\mathrm{b}-\mathrm{g}: n=10$. The value in b-g represent the mean \pm s.d. $p$ values from the student's $t$-test of the improved line against Kongyu131 is indicated. KY131 is the abbreviation of Kongyu 131

100-grain weight increased significantly. The result manifests that the improved lines' grain length and 100-grain weight increased significantly compared with Kongyu 131. Thus, the method is efficient and accurate update the GS3 locus of Kongyu 131.

The allele of GKBR at GS3 locus can increase Kongyu 131 's grain length according to QTL analysis

To further confirm the allele of GKBR at GS3 locus can increase Kongyu 131's grain length, we made QTL analysis using $\mathrm{BC}_{3} \mathrm{~F}_{2}$ population constructed by Kongyu 131 and GKBR. We detected a QTL on chromosome 3 and the largest LOD score at the SNP3 (Fig. 5a), presumably this QTL is GS3 locus. Therefore, we confirmed that the allele of GKBR at GS3 can increase Kongyu 131's grain length (Figs. 5c, 6a).

\section{Discussion}

In this study, we applied the new approach updated the GS3 locus of Kongyu 131, and verified grain length and 100 -grain weight increased significantly of the improved line compared with Kongyu 131. So we convinced that the Update method is an accurate and efficient method to improve rice variety. First of all, the selection of target individual using the method is according to genotyping with SNP markers. Thus, the method overcomes the defects of time-consuming of traditional field selection by breeders' experience. Second, the selection of individual incorporated target gene by SNP marker within gene, so, this selection no concerns of omit target gene. Third, the selection process including not only forward selection but also background elimination. According to background elimination, on the one hand, it eliminate the effects of non-target segment, on the other hand, it reserves the background genome of recurrent parent at the most extent. More importantly, the way can update causal gene as soon as we find defects of the improved line. On the basis of above results, we convinced that the approach overcomes the defects of time-consuming, low predictability and reproducibility of traditional breeding. Thus, it is predictable that the way will be applied to improve crop to satisfy the increasing demand as an accurate and controlled and efficient method. 


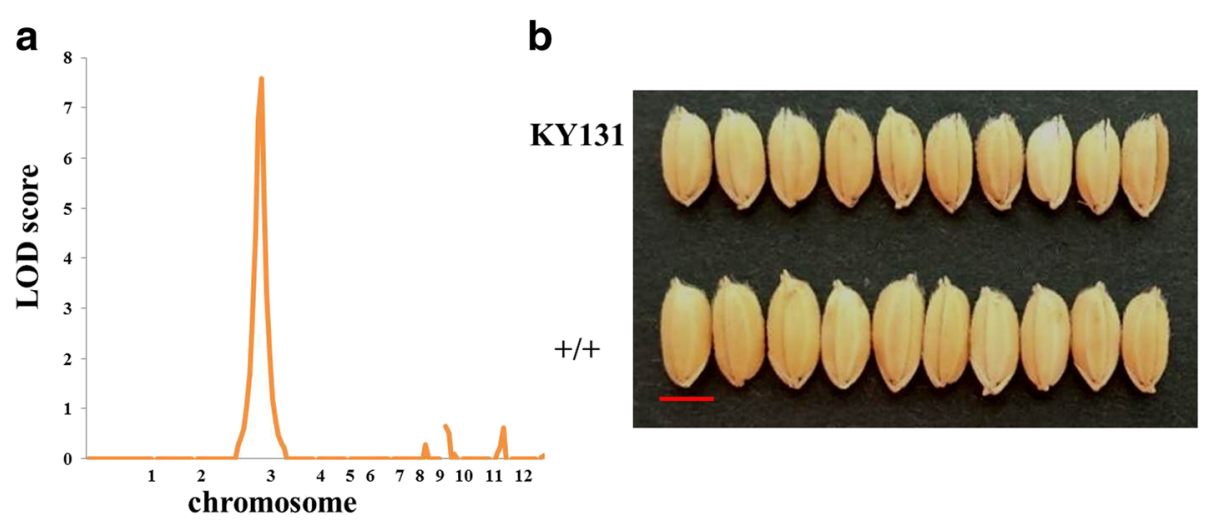

C

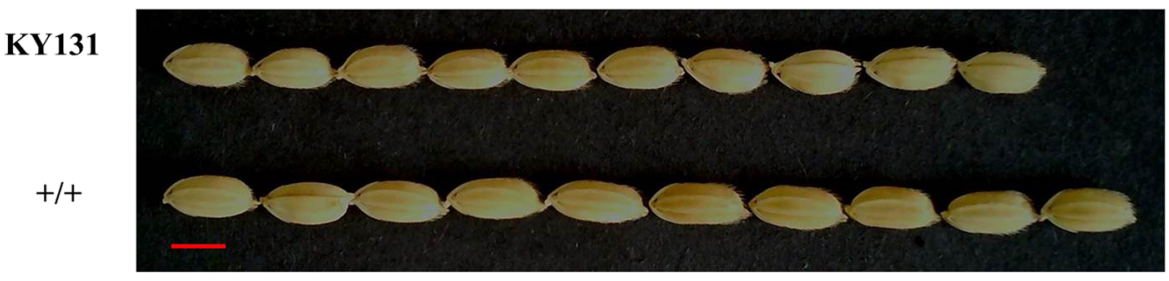

Fig. 5 QTL analysis confirmed that gs3 allele from donor GKBR increased grain length significantly. a QTL analysis using $\mathrm{BC}_{3} \mathrm{~F}_{2}$ population with 46 lines and 138 SNP markers. b, $\mathbf{c}$ the grain length is significantly increased with the donor GKBR allele at GS3 locus. Scale bar, $5 \mathrm{~mm}$. KY131 is the abbreviation of Kongyu 131

Meanwhile, we noticed that the total grain weight per plant of the improved line increased nonsiginificantly compared with Kongyu 131 when the planting density is $30 \mathrm{~cm} \times 20 \mathrm{~cm}$ and one plant per hill (Table 1). Also we find the total grain weight of the improved line increased siginificantly compared with Kongyu 131 when the planting density is $30 \mathrm{~cm} \times 20 \mathrm{~cm}$ and three to four plants per hill (Table 1). More importantly, the total grian weight of the improved line when the planting density is $30 \mathrm{~cm} \times 20 \mathrm{~cm}$ and three to four plants per hill increased more than when the planting density is $30 \mathrm{~cm} \times 14 \mathrm{~cm}$ and three to four plants per hill. Thus,

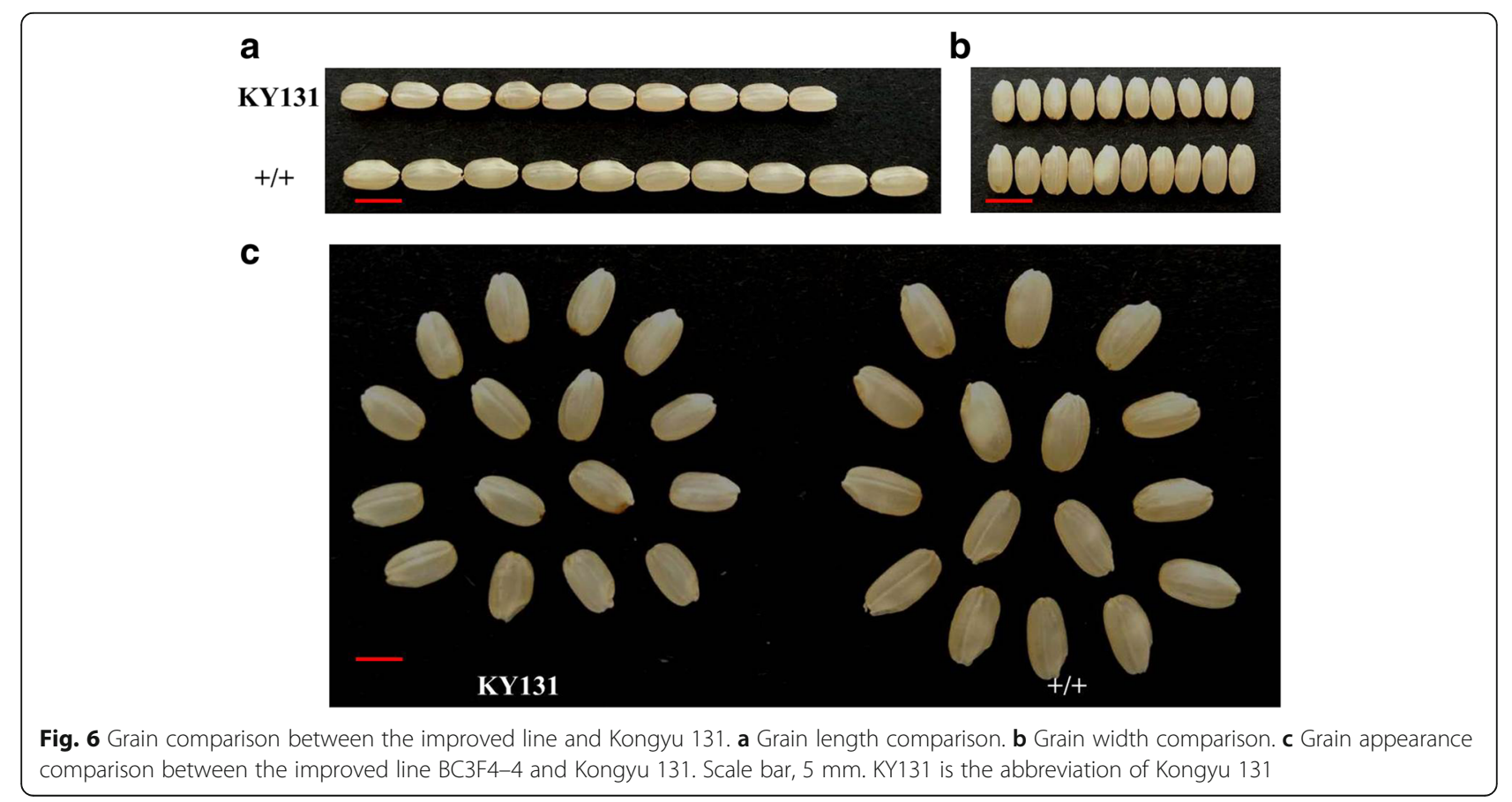


we believed that the total grain weight can increased significantly according to appropriate planting density. Next, we will verify the adapted planting density of the improved line by field experiment.

\section{Traditional breeding with MAS and update breeding}

The selection process of traditional breeding almost depends on field selection by experience of breeders. However, this selection has many inevitable defects including time-consuming, low predictability and reproducibility. Although marker-assisted selection (MAS) using markers on the basis of DNA sequence has greatly facilitate the accurate and diminish the time-cost of selection, unavoidable recombination limited precision of selection between target gene and markers, which mostly is linkage with target gene. But the selection process of Update breeding used in this study has no concern of omit target gene by using marker within gene.

Meanwhile, we designed four SNP markers anchored upstream and downstream of target gene at the process of selection using the Update method; those four markers are used to eliminate the drag of non-target segments surrounding the target gene. This process cannot achieve by using MAS, no mention to field selection process.

The traditional breeding selection and MAS have no consideration of whole genome scanning, namely background elimination, except select for target phenotype. But the Update breeding selection including forward selection and background elimination scanning whole genome. According to background elimination it not only eliminates the effects of nontarget segment but also reserves the background of recurrent parent at the most extent.

According to reserve the background of the recurrent parent, the method resolved the defect that the traditional breeding hard to reusing the genome information. On the basis of whole genome sequence information, we can continuously detect and update causal gene lead to defects of the cultivar improved by the Update breeding. According to this process, the beneficial allele will accumulated within recurrent parent, and this can become a reality that cultivars updated by successively improving in the future, as computer software update.

As above mentioned, we can eliminate the non-target segment at chromosome 1 (Fig. 3d) by backcross with Kongyu 131 as soon as we found defects influenced by it at the cultivate process. This process of improvement reserves the background of the recurrent parent at the most extent.

\section{Genome information and update breeding}

We reckon that some elements must to be consideration to apply the Update breeding on the basis of above results and analysis. First, the prerequisite is the reliable and accurate genome sequence information. Second, also the thorough study of functional gene of many traits is a necessary foundation. Third, information management with high accuracy and efficient is vital necessity.

Many crops genome has sequenced or is sequencing with the development of sequencing technology. This information facilitates utilizing the Update breeding method in the future. However, there is much room to advance the accuracy of genome sequence information which will facilitate precision of whole genome elimination. And it is more efficient and accurate to update allele of specific trait using the Update breeding only when causal functional gene is definite. So it is vital to broad scope of identification and functional analysis of many genes. More importantly, management of information with accurate and efficient way is necessary to guarantee using that exactly.

With the development of exact genome sequence information and definite functional gene and high-efficiency information management, we hopefully believed that the Update breeding will be extensively applied to improve and update crops. And thus, it will satisfy the demand of increasing population all around the world.

\section{Conclusions}

To improve the grain size of an elite variety Kongyu 131, we carried out QTL analysis and detected desirable allele from donor GKBR around the GS3 locus. And we updated the grain size by introgressed the favorable allele from the donor using designed five SNP markers within and without GS3. Compared with Kongyu 131, grain length and 100-grain weight and total grain weight per plant of the improved line increased by $12.05 \%, 16.30 \%$ and $4.47 \%$, respectively. This result demonstrates that update the GS3 locus is a feasible and efficient and accurate way can be applied to improve grain size of rice.

\section{Methods}

\section{Parents and populations}

Kongyu 131, a small grain variety, used as the recurrent parent in this study. And the donor, GKBR, is an indica rice with longer grain while unable to be normal maturity in Heilongiiang province for inappropriate photoperiod and temperature conditions. GKBR was crossed with Kongyu 131 and the F1 was backcrossed with Kongyu 131 to produce a $\mathrm{BC}_{3} \mathrm{~F}_{1}$ population including 137 lines and so on. The large allele gs3 from GKBR was used in this study.

\section{Re-sequencing genome of two parents and sequence comparison of GS3}

We acquired the genome sequence and SNPs of the two parents of Kongyu 131 and GKBR by next generation 
sequencing (NGS) using HiSeq2000. And we downloaded GS3 sequence of Chuan 7 from database of NCBI (https://www.ncbi.nlm.nih.gov/), then made sequence comparison between Kongyu 131 and GKBR to verify the donor have large grain allele at GS3 locus.

\section{The function of GS3}

GRAIN SIZE 3(GS3) is the first molecularly characterized major QTL controls grain size and weight in rice. GS3 was identified from the progeny derived from a cross between Minghui 63 with large grain and Chuan 7 with small grain, which show remarkable difference in grain length and weight. According to map-based clone, the researchers found GS3 encodes a putative transmembrane protein containing a plant-specific organ size regulation (OSR) domain, a tumor necrosis factor receptor/nerve growth factor receptor (TNFR/NGFR) family cysteine-rich domain, and a von Willebrand factor type C (VWFC) domain (Fan et al. 2006; Mao et al. 2010; Zuo and Li 2014). The large grain variety, Minghui 63, has a base difference, which is adenine (A) compared with small grain variety, Chuan 7 with cytosine $(\mathrm{C})$ at the second exon. The single nucleotide mutation detected at the GS3 gene between these two different grain-length groups, which changed a cysteine codon (TGC) in the small-grain group to a termination codon (TGA) in the large-grain group. This nonsense mutation lead to premature termination of transcription at the long grain variety, indicating that long grain resulted from the loss of the function of the protein otherwise producing short grains (Fan et al. 2006).

\section{Development of SNP markers and genotyping}

We designed more than 219 SNP markers evenly distributed on the whole genome between Kongyu131 and GKBR based on SNPs from the re-sequenced data. Those markers are used to scanning whole genome, namely background elimination. Also we developed five SNP markers (Table 2)within and without GS3 locus used to forward selection. Those markers are confirmed by HRM analysis.

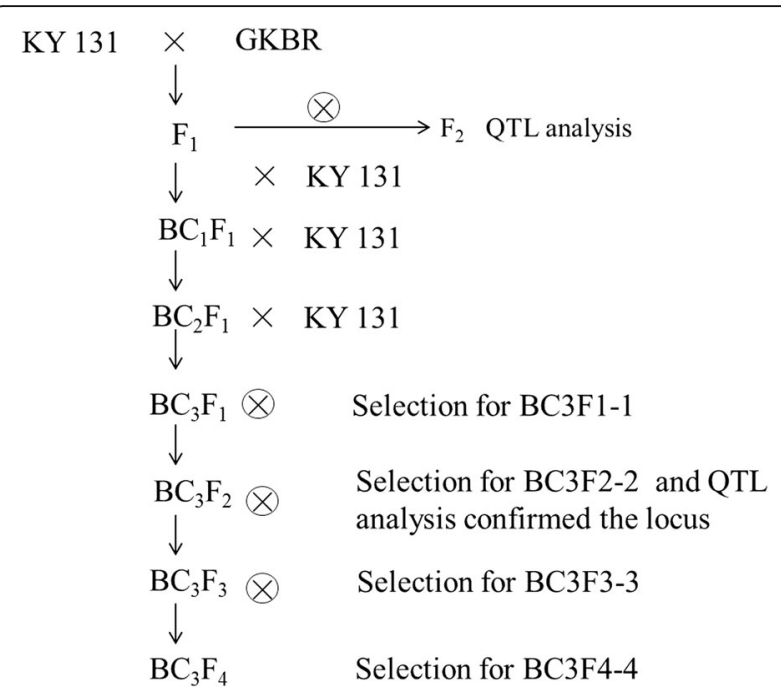

Fig. 7 Procedure of selection for the improved line BC3F4-4

\section{Selection for the improved line}

To gain the improved line incorporated the smallest segment at GS3 locus from donor GKBR, and also have the highest genetic background recovery ratio, at the beginning of selection, we selected individuals with heterozygote genotype at SNP3 from $\mathrm{BC}_{3} \mathrm{~F}_{1}$ population. And then, we used markers of SNP1-SNP5 select for individuals with recombination between SNP3 and SNP1 or SNP2 or SNP4 or SNP5. Namely, we selected individuals with recombination between SNP3 and its upstream and downstream at the same time. Finally, we selected an individual incorporated homozygote segment from donor GKBR at GS3 locus, and also with the highest genetic background recovery ratio (Fig. 7).

\section{The field plot trial identify the GS3's function of the improved line}

To verify GS3 function of the improved line, we cultivated Kongyu 131 and the improved line 96 individuals with three replications at Heilongjiang (130 $57^{\prime} \mathrm{E}, 46^{\circ} 23^{\prime}$ $\mathrm{N})$, respectively. According to general management of field, we investigated traits including grain length, grain width, 100-grain weight, plant height and total grain weight.

Table 2 Five SNP markers used to selection target chromosome segment

\begin{tabular}{lllll}
\hline Markers & Chr. & Position & Forward primer & Reverse primer \\
\hline SNP1 & 3 & $16,854,214$ & TGGTACACAGCATATCATGGAAC & CAGAAGTGTTATAACTACATATTTGC \\
SNP2 & 3 & $17,296,672$ & ATCTGCAACAAACAAGAGGATC & CTTGAGTTTCCACTCACAAACTTTC \\
SNP3 & 3 & $17,369,402$ & GAAACAGCTGGCTGGCTTACTCTC & GATCCACGCAGCCTCCAGATGC \\
SNP4 & 3 & $17,413,766$ & TTAGGACATATCGGCGTGCGTTA & CAGAGAAGCATCATTGAACGAACA \\
SNP5 & 3 & $17,874,647$ & ATGCAACCTITTCTCCCTTCCTAT & TCTAAAGGTTAACTCAGTAAAATCCT \\
\hline
\end{tabular}




\section{Abbreviations}

GS3: Grain size 3; HRM: High-resolution melting; MAS: Marker-assisted selection; QTL: Quantitative trait loci; SNP: Single nucleotide polymorphism

\section{Acknowledgments}

The authors would like to thank all members of Lin lab for kindly providing help.

\section{Funding}

This work was funded by the "Strategic Priority Research Program" of the Chinese Academy of Sciences (No.XDA08030102-1) and Talent Introduction Subject of State Key Laboratory of Plant Genomics, Institute of Genetics and Developmental Biology, University of Chinese Academy of Sciences (2014C0227-01)

\section{Availability of data and materials}

All data supporting the conclusions of this article are provided within the article.

\section{Authors' contributions}

SYL conceived the experiments. JZN carried out the most of the experiments, performed the statistical analysis and wrote the manuscript. CW, XMF, RSW, XHZ participated in primers design. GQJ, QBY and JXL performed rice hybridization and field management. All authors read and approved the final manuscript.

\section{Ethics approval and consent to participate}

Not applicable

\section{Competing interests}

The authors declare that they have no competing interests.

\section{Publisher's Note}

Springer Nature remains neutral with regard to jurisdictional claims in published maps and institutional affiliations.

Received: 8 December 2017 Accepted: 4 April 2018

Published online: 10 April 2018

\section{References}

Andersen JR, Lubberstedt T (2003) Functional markers in plants. Trends Plant Sci 8:554-560

Fan C, Xing Y, Mao H, Lu T, Han B, Xu C, Li X, Zhang Q (2006) GS3, a major QTL for grain length and weight and minor QTL for grain width and thickness in rice, encodes a putative transmembrane protein. Theor Appl Genet 112: 1164-1171

Feng X, Wang C, Nan J, Zhang X, Wang R, Jiang G, Yuan Q, Lin S (2017) Updating the elite rice variety Kongyu 131 by improving the Gn1a locus. Rice (N Y) 10:35

Haixiang H, Qian Q (2017) Progress in genetics research of rice grain shape and breeding achievements of long-grain shape and good quality japonica rice. Chin J Rice Sci 31:665-672

Huang R, Jiang L, Zheng J, Wang T, Wang H, Huang Y, Hong Z (2013) Genetic bases of rice grain shape: so many genes, so little known. Trends Plant Sci 18:218-226

James MG, Denyer K, Myers AM (2003) Starch synthesis in the cereal endosperm. Curr Opin Plant Biol 6:215-222

Knapp SJ (1998) Marker-assisted selection as a strategy for increasing the probability of selecting superior genotypes. Crop Sci 38:1164-1174

Lincoln SE, Daly MJ, Lander ES (1993) Constructing Genetic Linkage Maps with MAPMAKER/EXP Version 3.0. A Whitehead Institute for Biomedical Research Technical Report. Whitehead Institute, Cambridge

Mao H, Sun S, Yao J, Wang C, Yu S, Xu C, Li X, Zhang Q (2010) Linking differential domain functions of the GS3 protein to natural variation of grain size in rice. Proc Natl Acad Sci U S A 107:19579-19584

Miura K, Ashikari M, Matsuoka M (2011) The role of QTLs in the breeding of highyielding rice. Trends Plant Sci 16:319-326

Peleman JD, van der Voort JR (2003) Breeding by Design. Trends Plant Sci 8 : 330-334

Ray DK, Ramankutty N, Mueller ND, West PC, Foley JA (2012) Recent patterns of crop yield growth and stagnation. Nat Commun 3:1293
Sakamoto T, Matsuoka M (2008) Identifying and exploiting grain yield genes in rice. Curr Opin Plant Biol 11:209-214

Takeda S, Matsuoka M (2008) Genetic approaches to crop improvement: responding to environmental and population changes. Nat Rev Genet 9:444-457

Wang Y, Li J (2008) Molecular basis of plant architecture. Annu Rev Plant Biol 59: $253-279$

Wang Y, Li J (2011) Branching in rice. Curr Opin Plant Biol 14:94-99

Wittwer CT, Reed GH, Gundry CN, Vandersteen JG, Pryor RJ (2003) Highresolution genotyping by amplicon melting analysis using LCGreen. Clin Chem 49:853-860

Xing Y, Zhang Q (2010) Genetic and molecular bases of rice yield. Annu Rev Plant Biol 61:421-442

Zhou SR, Yin LL, Xue HW (2013) Functional genomics based understanding of rice endosperm development. Curr Opin Plant Biol 16:236-246

Zuo J, Li J (2013) Molecular dissection of complex agronomic traits of rice: a team effort by Chinese scientists in recent years. Natl Sci Rev 1:253-276

Zuo J, Li J (2014) Molecular genetic dissection of quantitative trait loci regulating rice grain size. Annu Rev Genet 48:99-118

\section{Submit your manuscript to a SpringerOpen ${ }^{\circ}$ journal and benefit from:}

- Convenient online submission

- Rigorous peer review

- Open access: articles freely available online

- High visibility within the field

- Retaining the copyright to your article

Submit your next manuscript at $>$ springeropen.com 